\title{
Criminologie
}

\section{La détention provisoire et sa mesure}

\section{Pierre Tournier}

Volume 28, numéro 2, 1995

La détention provisoire

URI : https://id.erudit.org/iderudit/017371ar

DOI : https://doi.org/10.7202/017371ar

Aller au sommaire du numéro

Éditeur(s)

Les Presses de l'Université de Montréal

ISSN

0316-0041 (imprimé)

1492-1367 (numérique)

Découvrir la revue

\section{Citer cet article}

Tournier, P. (1995). La détention provisoire et sa mesure. Criminologie, 28(2), 27-41. https://doi.org/10.7202/017371ar

\section{Résumé de l'article}

Considering debates frequently raised in France concerning the situation of pre-trial detention, the author identifies some trends: the constant reference to statistics; lack of efforts to precise the meaning of indicators used in making a demonstration - in fact the same statistics can be used to demonstrate contradictory theses -, a largely spread habit to always speak of more : more use of pre-trial incarceration, more pre-trial detainees... Those practices, argue the author, lead to ignore important changes in trends and to avoid questioning the meaning of those. The limited interest in research using more sophisticated indicators - that could add usefully informations to the data published regularly by the prison administrations -, international comparisons between data not necessarily comparable, references to old statistics, all result in everyone continuing to attribute to France the European championship in terms of pre-trial detention, while the actual situation could be totally different. Considering all those elements, the author presents new bases to reanimate the debate on the question of the use of pre-trial detention.
Ce document est protégé par la loi sur le droit d'auteur. L'utilisation des services d’Érudit (y compris la reproduction) est assujettie à sa politique d'utilisation que vous pouvez consulter en ligne.

https://apropos.erudit.org/fr/usagers/politique-dutilisation/ 
Considering debates frequently raised in France concerning the situation of pre-trial detention, the author identifies some trends: the constant reference to statistics; lack of efforts to precise the meaning of indicators used in making a demonstration - in fact the same statistics can be used to demonstrate contradictory theses -, a largely spread habit to always speak of more: more use of pre-trial incarceration, more pre-trial detainees... Those practices, argue the author, lead to ignore important changes in trends and to avoid questioning the meaning of those. The limited interest in research using more sophisticated indicators - that could add usefully informations to the data published regularly by the prison administrations - international comparisons between data not necessarily comparable, references to old statistics, all result in everyone continuing to attribute to France the European championship in terms of pre-trial detention, while the actual situation could be totally different. Considering all those elements, the author presents new bases to reanimate the debate on the question of the use of pre-trial detention.

Comme bien des débats concernant le fonctionnement du système de justice pénale, ceux qui portent sur la détention provisoire ont souvent recours aux statistiques ou, plus généralement, aux nombres. Dans l'ensemble des démocraties occidentales, l'objectif est de réduire, autant que faire se peut, l'usage de la détention provisoire, sorte de mal nécessaire. Aussi faut-il être en mesure d'évaluer son importance numérique, son évolution dans le temps et dans l'espace international et, si possible, les raisons des écarts observés. Nous voudrions revenir, dans cet article, sur les différents indicateurs généralement utilisés sans que parfojs on en connaisse la définition précise : "taux de prévenus", "taux de détention provisoire ", "taux de prévenus avant jugement», etc. Pour cela, nous ferons appel à trois bases de données que nous avons constituées et qui permettront, grâce à leur complémentarité, d'aborder les principaux aspects du sujet.

1. L'auteur est ingénieur de recherche au Centre de recherches sociologiques sur le droit et les institutions pénales (C.E.S.D.I.P.), expert scientifique au Conseil de l'Europe et chargé de cours à l'Institut de démographie de l'Université Paris I Pantheon-Sorbonne. Son adresse est : C.E.S.D.I.P., Immeuble Edison, 43 boul. Vauban, Gúyancourt 78280 France. 
Nous répondrons à la question «qu'est-ce que la détention provisoire? ", de façon concrète en prenant l'exemple des statistiques produites par l'administration pénitentiaire française. Sérialisées par notre soin (base SEPT), ces données statistiques administratives seront complétées par les résultats d'une recherche du CESDIP reposant sur l'observation suivie d'une cohorte d'entrants en prison. Enfin, nous examinerons les dernières données que nous avons produites dans le cadre de la statistique pénale annuelle du Conseil de l'Europe (base SPACE).

Seul un usage rigoureux et une mise en perspective diachronique et synchronique de ces données permettent d'apporter aux débats sur la détention provisoire des éléments utiles.

\section{COMBIEN DE PRÉVENUS EN FRANCE?}

Le recensement de la population des "prévenus » dans les établissements pénitentiaires français est un sujet de polémique récurrent. À l'occasion de chaque réforme de la procédure pénale, et chacun sait qu'elles sont fréquentes (Robert, 1992), se repose la «question des statistiques».

Au $1^{\mathrm{er}}$ octobre 1994, la population carcérale de la métropole comprend 51301 détenus : 21391 prévenus, 29628 condamnés et 282 détenus soumis à une contrainte par corps («dettiers »); soit une proportion de prévenus de $42 \%$. C'est ce que l'on appelle habituellement le «taux de prévenus ».

Dans la statistique pénitentiaire, la distinction entre "prévenus» et " condamnés" repose sur l'article D.50 du Code de procédure pénale selon lequel «sont désignés par le mot condamnés uniquement les condamnés ayant fait l'objet d'une décision ayant acquis le caractère définitif. Toutefois [...] le délai d'appel accordé au Procureur général [...] n'est pas pris en considération à cet égard ».

De ce fait, la catégorie "prévenus " recouvre l'ensemble des détenus qui n'ont pas fait l'objet d'une condamnation définitive - à l'exception des "dettiers" assiınilés ici aux condamnés. Aussi est-il erroné de parler à propos des "prévenus", comme on le fait souvent, de «détenus en attente de jugement », puisque certains d'entre eux ont déjà fait l'objet d'un jugement en première instance (détenus en appel ou pourvoi). Il est encore plus fallacieux d'assimiler «détention provisoire » et «instruction» car c'est oublier que la durée de la détention provisoire, et donc le nombre de "prévenus » détenus à un instant donné, dépend aussi des délais d'audiencement, de jugement et d'examen des voies de recours.

Au $1^{\text {er }}$ octobre 1994, la population des «prévenus » se décompose de la façon suivante : 


\section{Tableau 1}

Structure juridique de la population des prévenus (métropole $1^{\text {er }}$ octobre 1994)

Ensemble des prévenus

Détenus en cours d'instruction

Détenus dont l'instruction est terminée,

en attente de jugement

Détenus faisant l'objet d'une procédure de comparution immédiate en attente de jugement :

Détenus en appel ou pourvoi ou dans les délais pour le faire

\section{2) 391}

$100 \%$

15139

$70,8 \%$

3079

$14,4 \%$

$9 ! 3$

$4,3 \%$

2260

$10,6 \%$

Source : base de données SEPT (CESDIP)

Ainsi, les prévenus en attente d'un jugement de première instance représentent $89 \%$ des « prévenus » et $37 \%$ de l'ensemble des détenus.

\section{Le changement de tendance de 1985}

Depuis 1970, le nombre de prévenus n'a pratiquement jamais cessé de progresser : de 10899 au $1^{\text {er }}$ janvier 1970 à 22060 au $1^{\text {er }}$ janvier 1985. De plus, cette augmentation est allée en s'accélérant : accroissement annuel moyen de $3,4 \%$ pour la période $1970-1974,4,2 \%$ pour $1975-1979$ et $6,8 \%$ pour 1980-1984. Mais après une hausse de près de $10 \%$ en 1984 , le nombre de prévenus tend à se stabiliser autour de 20000 .

\section{Tableau 2}

Évolution du nombre de prévenus au $1^{\text {er }}$ janvier (métropole) : moyenne quinquennale

\begin{tabular}{rrrrr}
\hline $70: 10899$ & $75: 12889$ & $80: 15849$ & $85: 22060$ & $90: 19909$ \\
$71: 10905$ & $76: 12825$ & $81: 17313$ & $86: 21146$ & $91: 19047$ \\
$72: 12813$ & $77: 13065$ & $82: 15274$ & $87: 21411$ & $92: 19550$ \\
$73: 12258$ & $78: 13820$ & $83: 17643$ & $88: 20251$ & $93: 20101$ \\
$74: 12023$ & $79: 14167$ & $84: 20080$ & $89: 19526$ & $94: 20026$ \\
$\mathrm{~m}: 11780$ & $: 13353$ & $: 17232$ & $: 20879$ & $: 19727$ \\
\hline
\end{tabular}

Source : base de données SEPT (CESDIP)

Ce changement de tendance coöncide avec la mise en application, à compter du $1^{\text {er }}$ janvier 1985 , de la loi du 9 juillet 1984 sur la détention provisoire qui institue le débat contradictoire: toute décision de mise en 
détention provisoire doit être précédée d'un débat auquel participent le juge d'instruction, un représentant du parquet et le mis en cause assisté de son avocat. Toutefois, la proportion de débats contradictoires ne débouchant pas sur une mise en détention est très faible (de l'ordre du dixième), et ne cesse de réduire. Ce n'est donc pas le débat en lui-même qui a diminué le nombre de détentions provisoires. On peut plutôt supposer que la complication supplémentaire engendrée par cette loi a incliné les parquets à restreindre le nombre d'affaires mises à l'instruction (Robert, 1992).

Mais il se pourrait que cette stabilisation soit de courte durée. Au cours des années 1985-1990, les entrées de prévenus n'ont pratiquement cessé de baisser. De plus, depuis 1986, on a observé une légère érosion de la durée des détentions provisoires, qui avait connu une augmentation continue depuis 1970. En revanche, les entrées de prévenus augmentent de nouveau en 1991 puis en 1992 (tableau 3). Mais on retrouve en 1993 un niveau d'entrées et de durée voisin de celui de 1990.

Tableau 3

Évolution du nombre d'entrées de prévenus et de l'indicateur de la durée moyenne de détention provisoire (métropole)

\begin{tabular}{ccc}
\hline & Entrées de prévenus & $\begin{array}{c}\text { Durée moyenne de } \\
\text { détention provisoire } \\
\text { (en nombre de mois) }\end{array}$ \\
\hline 1970 & 61441 & 2,1 \\
1975 & 65143 & 2,4 \\
1980 & 68947 & 2,9 \\
1984 & 72316 & 3,4 \\
1985 & 66332 & 3,9 \\
1986 & 67727 & 3,8 \\
1987 & 65181 & 3,8 \\
1988 & 64804 & 3,7 \\
1989 & 64027 & 3,7 \\
1990 & 61216 & 3,8 \\
1991 & 66034 & 3,5 \\
1992 & 69861 & 3,4 \\
1993 & 62098 & 3,9 \\
\hline
\end{tabular}

Source : base de données SEPT (CESDIP)

Ces données, produites régulièrement par l'administration pénitentiaire, peuvent être utilement complétées par les résultats d'une recherche qui reposait sur l'observation suivie d'une cohorte d'entrants (Barre, Tournier, 1988). 


\section{Suivi d'une cohorte d'entrants}

L'analyse concerne un échantillon représentatif des incarcérations de 1983. Sur les 1326 dossiers examinés, on compte $95 \%$ de libérations dans un délai de 26 à 27 mois, $50 \%$ des détentions s'achevant dans un délai de 2 mois et demi (en moyenne). Les temps de détention peuvent être décomposés en détention en tant que prévenu et détention en tant que condamné (ou dettier). Cette décomposition fait nécessairement appel à des choix hiérarchisés lorsque plusieurs motifs d'écrou justifient un même temps de détention (dossier à affaires multiples). Ainsi avons-nous fait primer le statut de "condamné " sur celui de "prévenu ", conformément à l'article D.52 du Code de procédure pénale. Une telle décomposition selon le statut pénal ne peut évidemment être faite que sur des détentions achevées. Ainsi, $50 \%$ des journées de détention se déroulent en détention provisoire. Par ailleurs, $76 \%$ des détentions comptent une part de détention provisoire; $50 \%$ ont une détention provisoire inférieure à 1,5 mois, et la durée moyenne de détention provisoire est de 2,4 mois.

Comment s'inscrivent ces durées de détention par rapport à l'ensemble de la détention? En d'autres termes, la détention provisoire couvre-t-elle la totalité de la détention ou, au contraire, n'en est-elle qu'une partie, et dans quelle proportion? Nous avons déjà vu qu'au total, $50 \%$ des journées de détention étaient des journées de détention provisoire. Cette proportion est d'autant plus forte que la détention totale est brève. Sur l'ensemble des détentions, on constate que $42 \%$ ont été constituées uniquement de détention provisoire. Mais $75 \%$ des détentions inférieures à 15 jours et $59 \%$ des détentions de 15 jours à moins d'un mois ne sont considérées que comme de la détention provisoire. Pour l'ensemble des détentions supérieures à un an (et inférieures à 27 mois), la proportion est de $8 \%$.

De plus, nous avons décomposé le temps de détention provisoire en fonction de deux critères, «nature juridique » et «imputation » sur un temps d'exécution de peine. L'essentiel de la détention provisoire étudiée tient au temps de l'instruction correctionnelle. 
Tableau 4

Entrants de 1983 :

décomposition de la détention provisoire selon la nature juridique

\begin{tabular}{lc}
\hline & $\begin{array}{c}\text { Journées de détention } \\
\text { provisoire en \% }\end{array}$ \\
Procédure rapide & $5,4 \%$ \\
Opposition & $0,4 \%$ \\
Instruction correctionnelle & $62,1 \%$ \\
Attente de jugement correctionnel & $15,4 \%$ \\
Instruction criminelle et attente de jugement & $9,4 \%$ \\
Appel, pourvoi & $7,3 \%$ \\
Ensemble & $100,0 \%$ \\
\hline
\end{tabular}

Source : Barre, Tournier, 1988

Le temps de détention provisoire acquiert a posteriori un caractère différent selon qu'une condamnation viendra en partie ou totalement lui donner valeur d'exécution de peine. Si cette condamnation n'intervient pas pendant le temps du suivi, le caractère de la détention provisoire nous reste inconnu, c'est ce que l'on appelle de la détention «à imputation inconnue ». Dans le cas contraire, on peut savoir si et dans quelle mesure la durée de la détention provisoire est imputée sur une durée de peine prononcée :

- $49 \%$ des détentions provisoires comportent du temps de détention à imputation inconnue, du fait d'une ordonnance de mise en liberté (décision de libération en cours d'instruction). Ce temps représente $38 \%$ du total de la détention provisoire.

- $7 \%$ des détentions provisoires comportent du temps non imputé, ce qui représente un peu moins de $3 \%$ du temps de détention provisoire.

Enfin, l'analyse des modalités de sortie montre que $36 \%$ des détentions se terminent par une ordonnance de mise en liberté (39\% si l'on se limite aux dossiers comprenant une seule affaire). Ces proportions sont encore plus importantes pour les mineurs : respectivement $78 \%$ et $82 \%$. La plupart de ces mineurs sont donc sortis de prison sans avoir été jugés. Pour cette sous-cohorte, le suivi a été mené au-delà de la libération, grâce à l'examen du casier judiciaire (Tournier, 1991 - pour les majeurs, l'analyse est en cours).

\section{Suivi, au-delà de la libération, d'une cohorte de mineurs}

Considérant l'ensemble des mineurs incarcérés en février 1983 et sortis du fait d'une ordonnance de mise en liberté, nous avons cherché à répondre aux questions suivantes: l'affaire a-l-elle été ultérieurement 
sanctionnée par une condamnation? Si oui, quelle a été la nature de la peine prononcée? Enfin, dans le cas où la sanction comporte une partie d'emprisonnement ferme, existe-t-il une relation entre le quantum prononcé et la durée de la détention provisoire effectuée?

Pour connaître la suite donnée à l'affaire qui avait motivé l'incarcération de février 1983, nous avons examiné l'état du casier au $1^{\text {er }}$ juillet 1988. La plupart des mineurs concernés sont restés moins de six mois en détention $(97 \%)$. Ce qui donne un délai entre la libération et la date d'examen du casier de l'ordre de 5 ans. Compte tenu du délai qui s'écoule entre la date de la condamnation et la date de son inscription au casier judiciaire, les condamnations les plus tardives ne peuvent évidemment pas être recensées de manière exhaustive. Mais la longueur du délai d'observation choisi permet de penser que ce biais est certainement de faible ampleur.

Les mineurs libérés du fait d'une ordonnance de mise en liberté, et dont la détention était liée à une seule affaire, se répartissent selon la décision prise après leur libération de la manière suivante :

- $28 \%$ n'ont pas été condamnés (pas d'inscription au casier);

- $29 \%$ ont fait l'objet d'une condamnation sans prison ferme;

$\begin{array}{lll}\text { - mesure éducative } & : & 3 \% \\ \text { - amende } & : & 1 \% \\ \text { - mesure de substitution } & : & 1 \% \\ \text { - emprisonnement avec sursis total } & : & 24 \%\end{array}$

- $43 \%$ ont été condamnés à l'emprisonnement ferme.

Ainsi pour une majorité d'entre eux $(57 \%)$, la détention provisoire n'a pas été suivie d'une condamnation à l'emprisonnement ferme.

Les mineurs condamnés ont été jugés dans un délai moyen d'un an après leur libération, $37 \%$ d'entre eux étant condamnês plus d'un an après la levée d'écrou. Par ailleurs, il s'est écoulé, en moyenne, 15 mois entre les faits et la condamnation, ce délai étant supérieur à un an dans $47 \%$ des cas.

La proportion de non-condamnés qui, on l'a vu, est globalement de $28 \%$, décroît lorsque la durée de détention provisoire augmente, de $37 \%$ lorsque la détention est de « $5-10$ jours » à $22 \%$ lorsque la durée est de « 6 mois ou plus». Il existe une exception à cette « règle », les très courtes détentions ("moins de 5 jours") pour lesquelles on trouve la plus faible proportion de non-condamnés $(16 \%)$.

Parmi les condamnés, $60 \%$ ont fait l'objet d'une peine d'emprisonnement ferme (avec ou sans sursis partiel). Cette proportion augmente considérablement avec la durée de détention provisoire: de $27 \%$ après une détention de «moins de 5 jours » à $82 \%$ pour les détentions de « 1 à moins 
de 6 mois " (voire $100 \%$ pour les durées de « 6 mois et plus », mais l'effectif de référence de cette catégorie est faible).

Un tiers des mineurs condamnés à une peine d'emprisonnement ferme (avec ou sans sursis partiel) ont subi une détention provisoire supérieure au quantum ferme de la peine prononcée. Ce taux global recouvre des situations très différentes selon la durée de la détention provisoire; il croît avec la durée de détention: de $3 \%$ pour les détentions de «moins de 10 jours » à $71 \%$ pour celles de « 3 mois et plus».

L'influence de la durée de la détention provisoire sur le quantum ferme prononcé apparaît encore plus nettement dans le tri croisé présenté ci-dessous. Ainsi, $70 \%$ des effectifs se trouvent sur la diagonale du tableau (détention provisoire et quantum prononcé dans la même tranche). Par ailleurs, la liaison est de plus en plus forte à mesure que la détention provisoire augmente.

Tableau 5

Durée de la détention provisoire et quantum ferme de la peine d'emprisonnement prononcée (mineurs)

\begin{tabular}{lrrrr}
\hline Détention provisoire $=$ & - de $\mathbf{1 0} \mathbf{j}$. & $\mathbf{1 0} \mathbf{a ̀ ~ 3 0 ~ j . ~}$ & $\mathbf{1}$ à $\mathbf{3} \mathbf{~ m}$. & $\mathbf{3} \mathbf{~ m . ~ e t ~ + ~}$ \\
\hline Quantum ferme prononcé & & & & \\
Moins de 10 jours & $\mathbf{5 0 , 0}$ & 1,9 & 0,0 & 0,0 \\
$10-30$ jours & 13,2 & $\mathbf{6 7 , 9}$ & 2,8 & 0,0 \\
$1-3$ mois & 21,0 & 17,0 & $\mathbf{8 0 , 5}$ & 0,0 \\
3 mois et plus & 15,8 & 13,2 & 16,7 & $\mathbf{1 0 0 , 0}$ \\
Ensemble & 100,0 & 100,0 & 100,0 & 100,0 \\
\hline
\end{tabular}

Source : Tournier, 1991

Si l'on considère l'ensemble des journées de détention provisoire effectuées par les mincurs libérés du fait d'une ordonnance de mise en liberté, on constate que $43 \%$ d'entre elles n'ont pas été imputées sur une peine : «pas de condamnation»: $26 \%$, «condamnation sans prison ferme »: $13 \%$, «quantum inférieur à la détention provisoire»: $4 \%$.

En résumé, l'étude du devenir des libérés sur ordonnance de mise en liberté conduit aux conclusions suivantes:

- plus la détention provisoire est longue, plus la proportion de condamnés est importante ;

- en cas de condamnation, plus la détention provisoire est longue plus la proportion de condamnés à l'emprisonnement ferme est importante ; 
- et dans ce dernier cas, le quantum prononcé est étroitement lié à la durée de la détention provisoire effectuée.

\section{Comparaisons internationales}

Les statistiques pénales du Conseil de l'Europe, produites depuis 1983, font appel, en matière de détention provisoire, à deux indices de nature différente mais reposant sur la même définition de la notion de prévenus : le taux de prévenus et le taux de détention provisoire.

Cette définition est analogue à celle qui est utilisée dans la statistique française : l'ensemble des détenus qui n'ont pas fait l'objet d'une condamnation définitive constitue la catégorie "prévenus». Défini par la négative, ce groupe recouvre donc des catégories légales qui vont varier d'un pays à l'autre. Malgré cela, cette dichotomie permet de bien distinguer, de façon homogène, les deux grandes catégories de détenus: d'une part, ceux qui purgent une peine et, d'autre part, ceux qui sont en attente d'une décision statuant sur leur sort.

Le taux de prévenus - on pourrait parler plus simplement de pourcentage de prévenus - obtenu en rapportant le nombre de prévenus à une date donnée au nombre total de détenus à la même date, fluctue considérablement d'un pays à l'autre (tableau 6).

Certaines populations carcérales sont presque exclusivement composées de condamnés (Finlande, Irlande, Islande), alors que dans d'autres cas, plus d'un détenu sur deux n'a pas encore fait l'objet d'une condamnation définitive. Avec un taux de prévenus de $39,1 \%$ au $1^{\text {er }}$ septembre 1993 (départements d'outre-mer inclus), la France arrive en sixième position après la Turquie $(56 \%)$, l'Italie $(54 \%)$, la Belgique (49\%), la Tchécoslovaquie $(47 \%)$ et la Roumanie $(41 \%)$.

Indicateur d'usage courant, le taux de prévenus présente l'inconvénient de dépendre à la fois du nombre des «prévenus " et des «condamnés ". Ainsi, l'augmentation de ce taux à la suite d'une amnistie ou d'une grâce collective signifie seulement une baisse du nombre de condamnés, sans modification de celui des prévenus. Plus significatif est le taux de détention provisoire, indice obtenu en rapportant, à une date donnée, le nombre de prévenus au nombre d'habitants. Il est généralement exprimé pour 100000 habitants. Au $1^{\text {er }}$ septembre 1993, ce taux est en France (départements d'outre-mer inclus) de 34 prévenus pour 100000 habitants, ce qui situe notre pays dans une position voisine de la Belgique (35), de l'Espagne (34), et du Luxembourg (35). Les taux sont plus élevés au Portugal (42), en Italie (48) et dans les pays de l'est européen : Pologne (39), République tchèque (78), Roumanie (81). 
Ainsi la situation de la France, en matière de détention provisoire au sein de l'Europe n'a pas - ou n'a plus - ce caractère exceptionnel souvent signalé. Mais, compte tenu de l'hétérogénéité de la catégorie "prévenus », il ne peut s'agir là que d'une première approche comparative.

L'introduction, en 1993, dans la statistique du Conseil de l'Europe, d'une nomenclature plus fine pour décrire le statut pénal des détenus a permis d'améliorer les éléments de comparaison.

Cette nomenclature est la suivante :

a. Détenus condamnés (condamnation définitive);

b. Détenus condamnés ayant utilisé une voie de recours ou qui sont dans les délais légaux pour le faire;

c. Détenus déclarés coupables, non encore condamnés;

d. Détenus non jugés (non encore déclarés coupables);

e. Autres cas.

À partir de cette nomenclature, nous avons calculé des taux de prévenus avant jugement et des taux de détention provisoire avant jugement pour 100000 habitants (tableau 8). Pour le calcul de ces taux, seuls sont pris en compte les détenus recensés dans la rubrique «détenus non jugés». La France apparaît très nettement en tête en ce qui concerne le taux de prévenus avant jugement. En revanche, en matière de taux de détention provisoire avant jugement pour 100000 habitants, indice plus significatif, comme nous l'avons vu ci-dessus, la France est dépassée par l'Espagne, la Pologne, la République tchèque, et l'Irlande du Nord.

\section{CONCLUSION}

En conclusion de cette brève présentation de la détention provisoire et des instruments de mesure disponibles dans les statistiques françaises et dans la statistique du Conseil de l'Europe (SPACE), nous soulignerons la principale lacune de ces bases de donnćcs : la non-prise en compte de la nature de l'infraction (ou des infractions) poursuivie(s). Dans la statistique française, cette lacune va bientôt être comblée, en partie, dans l'exploitation du Fichier national des détenus (FND). Pour les données européennes, nous avions proposé une nomenclature d'infractions permettant de connaître la répartition des entrées selon le motif. Il n'était pas envisageable de proposer une nomenclature trop fine. C'est pourquoi chaque délégation nationale trouvait à critiquer telle ou telle imprécision de la nomenclature ou encore la non-prise en compte, de façon explicite, de tel ou tel contentieux jugé important. Le projet fut abandonné. Il devait être repris sous une autre forme dans l'avenir car on ne peut pas se satisfaire de la situation présente dans ce domaine. 


\section{Tableau 6}

La détention provisoire dans les pays membres du Conseil de l'Europe Situation au 1.09.1993

\begin{tabular}{|c|c|c|c|c|c|}
\hline & & $\begin{array}{l}\text { Nombre total } \\
\text { de détenus }\end{array}$ & $\begin{array}{c}\text { Taux de } \\
\text { détention par } \\
100000 \\
\text { habitants }\end{array}$ & $\begin{array}{c}\text { Taux de } \\
\text { prévenus en \% }\end{array}$ & $\begin{array}{c}\text { Taux de } \\
\text { détention } \\
\text { provisoire par } \\
100000 \\
\text { habitants } \\
\end{array}$ \\
\hline Allemagne $(*)$ & $:$ & 65838 & 81,0 & 38,1 & 30,9 \\
\hline Autriche & : & 7099 & 91,0 & 33,9 & 30,8 \\
\hline Belgique & $:$ & 7203 & 72,1 & 48,7 & 35.1 \\
\hline Bulgarie $\left(^{*}\right)$ & $:$ & 8364 & 98,9 & 31,3 & 30,9 \\
\hline Chypre & $:$ & 188 & 30,0 & $\ldots$ & $\ldots$, \\
\hline Danemark & $:$ & 3702 & 71,0 & $\ldots$. & $\ldots$. \\
\hline Espagne & : & 45711 & 14,9 & 29,8 & 34,2 \\
\hline Finlande & $:$ & 3132 & 61,8 & 8,7 & 5,4 \\
\hline France & : & $51 \quad 134$ & 86,3 & 39,1 & 33,7 \\
\hline Grèce $\left(^{*}\right)$ & $:$ & 6524 & 68,0 & .... & $\ldots$. \\
\hline Hongrie & $:$ & 13196 & 132,0 & $\ldots$. & $\ldots$, \\
\hline Irlande & : & 2108 & 59,8 & 7,9 & 4,7 \\
\hline Islande & $:$ & 103 & 38,9 & 3,9 & 1,5 \\
\hline Italie & $:$ & 50794 & 89,0 & 54,0 & 48,0 \\
\hline Lituanie & : & 10324 & 275,0 & 38,1 & 104,9 \\
\hline Luxembourg & ; & 425 & 107,5 & 32,2 & 34,7 \\
\hline Norvège & $:$ & 2607 & 60,0 &... & $\ldots$ \\
\hline Pays-Bas & $:$ & 7843 & 51,0 & $\ldots$ & $\ldots$ \\
\hline Pologne & $:$ & 61895 & 160,0 & 24,1 & 38,5 \\
\hline Portugal & : & 10904 & 111,0 & 37,4 & 1,6 \\
\hline Rép. slovaque & : & 7221 & 136,0 & $\ldots$ & $\ldots$ \\
\hline Rép. tchèque & : & 16567 & 165,0 & 47,1 & 77,8 \\
\hline Roumanie & : & 46189 & 200,0 & 40,6 & 81,1 \\
\hline $\begin{array}{l}\text { Royaume-Uni } \\
\text { Angleterre et } \\
\text { Pays de Galles }\end{array}$ & : & 45633 & 89,0 & $\ldots$ & $\ldots$ \\
\hline Écosse & : & 5900 & 115,0 & 17,7 & 20,3 \\
\hline Irlande du Nord $\left({ }^{*}\right)$ & & 1902 & 118,0 & 25.6 & 30,2 \\
\hline Suède & $:$ & 5794 & 66,0 & 21,8 & 14,4 \\
\hline Suisse & : & 5627 & 81,0 & $\ldots$ & $\ldots$. \\
\hline Turquie & $:$ & 31304 & 51,6 & 55,9 & 28,9 \\
\hline
\end{tabular}

(*) Allemagne (30.9.93), Bulgarie (31.12.93), Grèce (1.1.93) Irlande du Nord (26.8.93). 
Tableau 7

Taux de détention provisoire (au $1^{\text {er }}$ septembre, pour 100000 habitants): évolution 1983-1993

\begin{tabular}{|c|c|c|c|c|c|c|}
\hline & 1983 & 1984 & 1985 & 1986 & 1987 & 1988 \\
\hline Allemagne & 26,2 & 23,8 & 22,1 & 20,5 & 18,8 & 19,0 \\
\hline Autriche & 27,2 & 26,5 & 25,8 & 23,6 & 22,7 & 18,1 \\
\hline Belgique & 36,3 & 34,8 & 34,0 & 31,8 & 33,8 & 33,2 \\
\hline Chypre & 1,1 & 4,0 & 1,9 & 2,9 & 4,2 & 3,1 \\
\hline Danemark & 16,2 & 14,3 & 16,2 & 17,5 & 16,5 & 17,1 \\
\hline Espagne & 13,2 & 20.6 & 27,9 & 30,0 & 30,2 & 33,2 \\
\hline Finlande & $\ldots$ & ..,. & 10,7 & 11,6 & 11,2 & 8,9 \\
\hline France & 35,3 & 37,3 & 35,7 & 38,6 & 38,7 & 35,9 \\
\hline Grèce & 14,3 & 9,5 & 8,7 & 10,2 & 10,7 & 12,1 \\
\hline Irlande & 3,8 & 3,1 & 4,0 & 2,9 & 3,1 & 2,9 \\
\hline Islande & 2,6 & 5,5 & 7,1 & 2,1 & 2,1 & 2,8 \\
\hline Italie & 53,9 & 54,1 & 45,1 & 37,9 & 34,9 & 29,8 \\
\hline Luxembourg & 21,3 & 22,2 & 25.2 & 32,9 & 28,9 & 28,5 \\
\hline Malte & 11,1 & 10,9 & 9,4 & 14,6 & 11,2 & 46,1 \\
\hline Norvège & 13,2 & 12,4 & 10,2 & 10,8 & 13,0 & 11,1 \\
\hline Pays-Bas & 11,2 & 12,3 & 11,4 & 13,8 & 13,3 & 15.9 \\
\hline Portugal & 21,9 & 28,5 & 32,3 & 33,5 & 34,3 & 27,8 \\
\hline $\begin{array}{l}\text { Royaume-Uni } \\
\text { Angleterre et }\end{array}$ & 16,9 & 18,6 & 20,4 & 20,9 & 21,2 & 20,0 \\
\hline Pays de Galles & 16,8 & 18,1 & 20,0 & 21,0 & 21,5 & 20,4 \\
\hline Suède & 8,1 & 8,6 & 8,3 & 9,2 & 10,1 & 11,2 \\
\hline Suisse & 20,4 & 24,0 & 15,5 & 17,0 &.,. & 23,8 \\
\hline Turquie & $\ldots$ & 69,1 & 49,1 & 42,6 & 37,7 & 36,4 \\
\hline
\end{tabular}

Source : SPACE (Conseil de l'Europe) 
Tableau 7 (suite)

\begin{tabular}{|c|c|c|c|c|c|}
\hline & 1989 & 1990 & 1991 & 1992 & 1993 \\
\hline Allemagne & 19,7 & 20,6 & 24,1 & $\ldots$. & 30,9 \\
\hline Autriche & 21,7 & 25,8 & 28,7 & 35,3 & 30,8 \\
\hline Belgique & 32,6 & 30,9 & 31,2 & 36,3 & 35,1 \\
\hline Chypre & 4,8 & 3,8 & $\ldots$ & $\ldots$, & .... \\
\hline Danemark & 19,1 & 16,7 & $\ldots$ & 19,2 & $\ldots$ \\
\hline Espagne & 33,0 & 33,8 & 32,4 & 30,5 & 34,2 \\
\hline Finlande & 6,9 & 7,2 & 5,8 & 5,7 & 5,4 \\
\hline France & 35,6 & 33,4 & 34,8 & 36,5 & 33,7 \\
\hline Grèce & 16,7 & ..,. & 17,2 & 21,7 & $\ldots$ \\
\hline Irlande & 3,2 & $\ldots$ & 3,9 & 4,2 & 4,7 \\
\hline Is!ande & 4,3 & 1,6 & 2,3 & 4,6 & 1,5 \\
\hline Italie & 24,4 & 23,0 & 29,6 & 45,7 & 48,0 \\
\hline Luxembourg & 20,2 & 24,6 & 18,2 & 24,7 & 34,7 \\
\hline Malte & $\ldots$ & ..,. & $\ldots$ &.., & $\ldots$ \\
\hline Norvège & $\ldots$. & 11,6 & 12,0 & ..,. & $\ldots$ \\
\hline Pays-Bas & 17,6 & 17,2 & $\ldots$, & $\ldots$. & ..., \\
\hline Portugal & 35,6 & 28,1 & 29,1 & 36,9 & 41,6 \\
\hline $\begin{array}{l}\text { Royaume-Uni } \\
\text { Angleterre et } \\
\text { Pays de Galles }\end{array}$ & 21,3 & 20,0 & 20,5 & $\ldots$ & $\ldots$ \\
\hline Suède & 10,9 & 11,7 & 12,1 & 12,9 & 14,4 \\
\hline Suisse & 26,0 & 29,9 & 37,9 & 22,7 & $\ldots$ \\
\hline Turquie & 33,5 & 30,7 & 26,7 & $\ldots$ & 28,9 \\
\hline
\end{tabular}

Source : SPACE (Conseil de l'Europe) 
Tableau 8

La détention provisoire avant jugement dans les pays membres du Conseil de l'Europe - Situation au 1.09.1993

\begin{tabular}{lccc}
\hline & & $\begin{array}{c}\text { Taux de prévenus avant } \\
\text { jugement en \% }\end{array}$ & $\begin{array}{c}\text { Taux de détention provisoire } \\
\text { avant jugement p. 100 000 }\end{array}$ \\
\hline Belgique & $:$ & 25,4 & 18,3 \\
Bulgarie & $:$ & 12,2 & 12,1 \\
Espagne & $:$ & 29,8 & 34,2 \\
France & $:$ & 34,5 & 29,8 \\
Irlande & $:$ & 7,9 & 4,7 \\
Islande & $:$ & 2,9 & 1,1 \\
Italie & $:$ & 30,6 & 27,2 \\
Luxembourg & $:$ & 23,1 & 24,8 \\
Pologne & $:$ & 23,6 & 37,7 \\
Rép. tchèque & $:$ & 24,6 & 40,5 \\
Royaume-Uni & & & \\
Angleterre et & & 18,9 & 16,8 \\
Pays de Galles & & 13,7 & 15,7 \\
Écosse & $:$ & 25,6 & 30.2 \\
Irlande du Nord & $:$ & 33,9 & 27,5 \\
Suisse & $:$ & & \\
\hline
\end{tabular}

Source : Tournier (sous presse) 


\section{RÉFÉRENCES BIBLIOGRAPHIQUES}

BARRE M.-D., TOURNIER P. (1988), La mesure du temps carcéral, observation suivie d'une cohorte d'entrants, CESDIP, Paris.

BARRE M.-D., TOURNIER P. (1990), «Le temps carcéral ", Revue de science criminelle et de droit pénal comparé, 2, pp. 379-387.

ROBERT Ph. Ed. (1992), Entre l'ordre et la liberté, la détention provisoire, deux siècles de débats, $\mathrm{L}$ 'Harmattan, Paris.

ROBERT Ph., TOURNIER P. (1993), «La détention provisoire », Revue de la gendar. merie nationale, 169 , pp. 13-16.

ROBERT Ph., AUBUSSON de CAVARLAY B., POTTIER M.-L, TOURNIER P. (1994), Les comptes du crime, les délinquances ef leurs mesures, L'HARMATTAN, Paris.

TOURNIER P., Base de données SEPT: séries pénitentiaires temporelles (données actualisées tous les ans).

TOURNYER P. (1991), La détention des mineurs, observation suivie d'une cohorte d'entrants, CESDIP, Paris.

TOURNIER P. (1992), Démographie des prisons françaises - toujours plus? CESDIP, Paris.

TOURNIER P. (1994), "Les sanctions alternatives à l'emprisonnement en Europe : synopsis ", in Le travail d'intérêt général 1984-1994, études et recherches, actes du Colloque pour le $X^{e}$ anniversaire du TIG en France, ministère de la Justice, pp. 11-20.

TOURNIER P. (1994), "The custodial crisis in Europe, - inflated prison populations and possible alternatives $-»$, Romainmôtier Lausanne, IV Colloquium on crime and criminal policy in Europe, European Journal on Criminal Policy and Research, vol. 2, 4, 89-10.

TOURNIER P., (sous presse), Statistique pénale annuelle du conseil de l'Europe (SPACE) : enquête 1993, Bulletin d'information pénologique, $n^{\circ} 19$. 